\title{
Desarrollo Turístico y Capacidades Colectivas en San Juan de Las Huertas, Zinacantepec, México
}

\author{
Tourism Development and Collective Capabilities in San Juan de Las \\ Huertas, Zinacantepec, México
}

Guadalupe Rodríguez Zermeño ${ }^{1}$

Lilia Zizumbo Villarreal ${ }^{2}$

\section{Resumen}

Los ejidatarios de San Juan de las Huertas, cuentan con recursos naturales como bosque, valles e infraestructura turística, pero no los han aprovechando de manera sustentable debido a que los ejidatarios no han consolidado formas de organización para el aprovechamiento de sus recursos, lo que ha provocado el deterioro de la infraestructura, de las áreas naturales y el escaso turismo en la zona. Las capacidades colectivas de la comunidad y específicamente de los ejidatarios no son suficientes para desarrollar proyectos que puedan beneficiarlos a largo plazo, es por ello que en el presente trabajo se dan a conocer los resultados del análisis de las capacidades colectivas de la comunidad, para el desarrollo del turismo.

Palabras-clave: desarrollo turístico; capacidades colectivas; desarrollo local; Zinacantepec; México.

\begin{abstract}
Ejidatarios de San Juan de las Huertas, natural resources like forests, valleys, and tourist infrastructure, but do not have a sustainable advantage because the farmers have not established forms of organization to use their resources, which has led to the deterioration of infrastructure, natural areas and poor tourism in the area. The collective capabilities of the community and specifically of the ejidatarios are not sufficient to develop projects that can benefit them long term, that is why in this paper we discuss the results of the analysis of the collective capabilities of the community, for tourism development.
\end{abstract}

Keywords: tourism development; collective capabilities; local development; Zinacantepec; México.

\footnotetext{
${ }^{1}$ Licenciada en Turismo por la Universidad Autónoma del Estado de México (UAEM), Maestra en Ciencias Ambientales por la UAEM, profesora de asignatura en la Facultad de Turismo y Gastronomía de la Universidad Autónoma del Estado de México, catedrática de Geografía Turística, Turismo Alternativo, Desarrollo Local y Regional, Impactos del Turismo en el Patrimonio Natural, Gestión del Patrimonio Natural, Teoría Sociológica y Turismo. E-mail: lupita_zermeno@hotmail.com

${ }^{2}$ Doctora en Sociología por la UNAM, profesora e investigadora de tiempo completo en la Facultad de Turismo de la Universidad Autónoma del México. Catedrática de Sociología del Turismo, Métodos y Técnicas para la investigación turística, Seminario de Tesis. E-mail: 1zv04@yahoo.com
} 


\section{Introducción}

Los orígenes del turismo en el ejido San Juan de las Huertas ubicado al sur del municipio de Zinacantepec, Estado de México, basado en la existencia de atractivos naturales (paisajes, valles intermontanos, atractivos geomorfológicos), se remontan a Mayo de 1975, fecha en que se concesiona al gobierno del Estado de México la construcción de un centro turístico El Parque de los Venados dentro del Parque Nacional Nevado de Toluca, mismo que contaba con instalaciones de una posada familiar y un albergue, la primera con 14 habitaciones dobles, agua caliente, sanitarios y equipo para cocinar. El segundo, con 36 literas y servicio sanitario, se dispone también de asadores, mesa bancos, área para juegos infantiles y otra para cautiverio de venado cola blanca, actualmente fuera de servicio. (Alberto 1997, Chávez y Cisneros 2004)

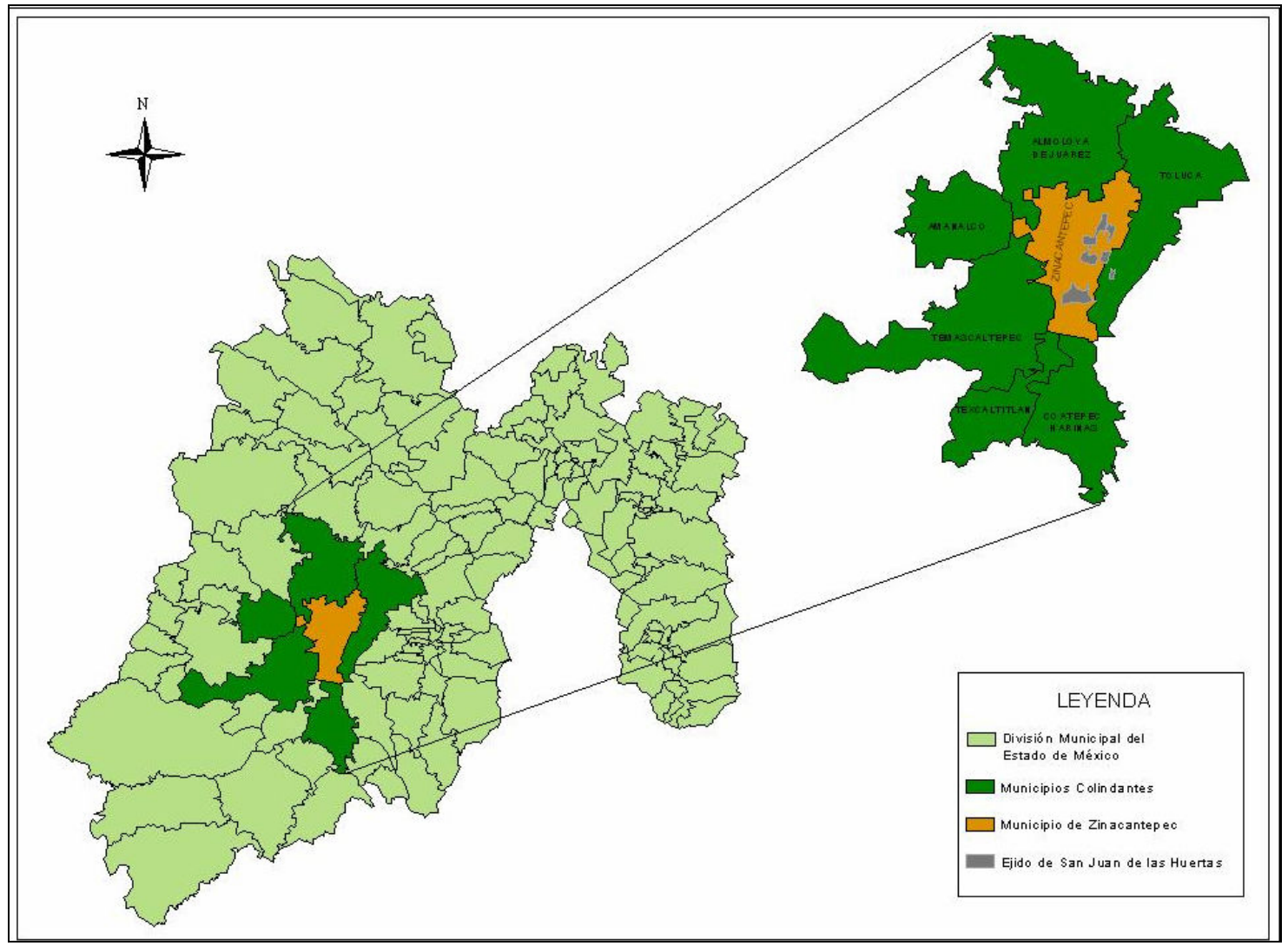

La administración de estas instalaciones ha estado a cargo de diversos sectores, en sus inicios por organismos públicos y ejidatarios. La posada familiar estuvo concesionada a una empresa privada, quedando finalmente bajo la responsabilidad de los ejidatarios de San Juan de las 
Huertas. En la actualidad por falta de mantenimiento adecuado y atención de personal capacitado presenta subutilización de su capacidad instalada. En particular el ejido no cuenta con infraestructura y servicios básicos, la forma como actualmente satisface los requerimientos de agua potable es a través de un manantial mediante la construcción de un depósito que abastece tanto al albergue como a la posada familiar.

En el parque no existen establecimientos de alimentos y bebidas, este servicio se restringe únicamente a puestos ambulantes y semifijos, los cuales se ubican en la desviación de la carretera No. 134 a lo largo de la tercería que conduce al cráter, en el Parque de los Venados, a la entrada al albergue y cerca de la caseta de acceso a las lagunas.

Lo anterior da cuenta de la reducida actividad turística en el Parque de los Venados, a pesar de contar con recursos naturales así como infraestructura turística, es por ello que en el presente trabajo se dan a conocer los resultados del análisis de las capacidades colectivas para el desarrollo del turismo en San Juan de las Huertas, siendo ésta, una comunidad ejidal la cual fue dotada de tierras con importantes recursos ricos en agua, bosque y áreas de cultivo, que al paso del tiempo éstas han sido insuficientes para la sobrevivencia lo que ha motivado el abandonado y explotación de manera irracional, generando conflictos por la forma en que se manejan los recursos.

De ahí que se plantearon las siguientes interrogantes ¿Por qué la comunidad de San Juan de las Huertas no ha podido consolidar el desarrollo turístico, cuando cuenta con recursos naturales e infraestructura para hacerlo? ¿Existe organización entre los miembros del ejido?, ¿Tienen la capacidad de generar proyectos y poder concretarlos?, ¿Tienen el interés por la actividad turística?

La investigación tuvo como objetivo analizar las capacidades colectivas que han posibilitado o limitado el desarrollo del turismo, así como la forma en que éste ha participado en el desarrollo local de San Juan de las Huertas. De igual forma se planteó como hipótesis que la actividad turística no se ha desarrollado, debido al debilitamiento de las capacidades colectivas de la comunidad, por tanto ésta no ha contribuido al desarrollo local de la comunidad.

La propuesta teórico metodológica que se siguió se sustenta en el análisis de las capacidades colectivas, el cual plantea que las capacidades colectivas comprenden tres tipos de capacidades, como lo es la capacidad organizacional, la cual permite a los miembros de una comunidad llegar a acuerdos que los beneficien y tengan un fin común, la segunda capacidad 
es la instrumental, que hace referencia a la habilidad para generar sus propios instrumentos tanto de planeación, de concertación, gestión y comunicación social; y finalmente la capacidad sistémica, misma que comprende el conocimiento de los actores locales en relación al sistema económico nacional e internacional así como al uso de nuevas tecnologías para promover su desarrollo.

La investigación se desarrolló en dos etapas, una documental y otra de campo. La primera, se baso en la identificación, revisión y acopio de información obtenida de libros, artículos, datos estadísticos, documentos oficiales. Los cuales fueron obtenidos de diferentes fuentes de información como bibliotecas de la Universidad Nacional Autónoma de México, la Universidad Autónoma del Estado de México (Facultad de Geografía, de Planeación Urbana y Regional y de Turismo), Instituto Nacional de Estadística Geografía e Informática (INEGI), Archivo Histórico de Toluca, y Registro Agrario del Estado de México. Esta información fue necesaria para identificar el espacio geográfico de la comunidad, sus recursos, caracterización de la población, datos estadísticos en relación a la organización ejidal así como los recursos con los que cuentan.

En la investigación de campo se aplicaron tres instrumentos, la encuesta se dirigió a los ejidatarios (dueños de los recursos naturales de la comunidad), de la que se obtuvo información cuali-cuantitativa con respecto a las capacidades de estos actores para el desarrollo de la actividad turística; la guía de entrevista, la cual permitió obtener información directa de informantes clave en este caso el presidente ejidal así como los integrantes del comisariado, quienes son los directamente involucrados con el Parque de los Venados y finalmente la guía de observación la cual permitió identificar las características del lugar, el grado de desarrollo de la actividad turística, así como la participación de los actores en la misma, con ellas se obtuvo información directa de la comunidad pudiendo tener un mayor acercamiento con la realidad, es así como identificar y analizar las capacidades de los actores locales y las condiciones reales para la práctica de la actividad turística dentro del Parque de los Venados.

Los instrumentos utilizados durante la investigación, nos permitieron recopilar información tanto cualitativa como cuantitativa, y a partir de ella se realizó un análisis de los mismos. El trabajo de campo se realizó en el periodo comprendido entre noviembre de 2007 y marzo de 2008, aplicando los diferentes instrumentos en veinte visitas a la comunidad, se aplicaron un total de 50 encuestas dirigidas a ejidatarios, el instrumento contó con 70 preguntas las cuales giraban en torno a medir las capacidades de dichos actores para el desarrollo de la actividad 
turística dentro del Parque de los Venados, como la capacidad organizacional, instrumental y sistémica; se realizaron 10 entrevistas a profundidad a informantes clave dentro del comisariado ejidal así como personas de la comunidad las cuales proporcionaron información con respecto al desarrollo de la comunidad así como las formas de organización de la misma, la participación de la población con el grupo ejidal y el desarrollo de la actividad turística en el Parque .

\section{Desarrollo Local}

El desarrollo se ha visualizado desde tres perspectivas, la primera como un proceso histórico por el cual todo país pasa, la segunda lo considera como un modelo de desarrollo y la tercera donde el desarrollo se ha visto como una política utilizada en los discursos por las autoridades (Preston, 1999). Algunos teóricos han abordado el desarrollo local desde una perspectiva económica más como crecimiento que como beneficios integrales de las poblaciones (Vázquez Barquero 2001, Boisier 2001, Vargas 2007), otros académicos proponen otros elementos que de alguna manera influyen en el desarrollo de una sociedad como la cultura, formas de organización (Arocena 2001, Coraggio 2003, Enriquez 1998, Marsiglia y Pintos 1997); cada sociedad ha tenido su propio proceso de desarrollo de acuerdo a sus condiciones políticas, sociales, culturales y económicas. Por lo tanto el desarrollo local no sólo tiene que ver con el aspecto económico sino que lo sociocultural es un elemento indispensable a considerar para que éste pueda tener éxito y con ello mejorar la calidad de vida de la población, ya que cuando se fomenta solo la organización para ejercer un recurso financiero se olvidan de la consolidación del proyecto y se piensa que con ello se mejorará la calidad de vida.

Los modelos de desarrollo no han funcionado adecuadamente, ya que al momento de ser copiados de países desarrollados a países subdesarrollados, éstos no se encuentran en las mismas condiciones, por tanto se vuelve inoperante el modelo. Con el neoliberalismo se pensó que podría dar respuesta a los principales problemas en los países subdesarrollados, sin embargo, no ha sido así, hoy se observa que éstas políticas han traído como consecuencia que los países ricos se vuelvan más ricos y los pobres cada vez son más pobres en lugar de mejorar las condiciones de vida de la población.

No se puede aplicar un modelo de desarrollo global donde se pretende unificar diferentes aspectos, debido a que cada país, región o comunidad cuenta con sus propias características 
económicas, sociales, culturales y políticas que lo hace único, por lo tanto, estos modelos generales no pueden ser aplicables porque no existen las mismas condiciones; por ejemplo el factor económico: las condiciones no son las mismas, no pueden responder de la misma forma aquellas localidades que cuentan con una base económica sólida a aquellas comunidades que no han logrado desarrollar alguna actividad económica, por lo tanto su competitividad no puede ser la misma, ni pueden responder de la misma manera ante un mundo global.

En el nuevo modelo económico se puede observar el capitalismo de manera global donde su principal preocupación, que va en el sentido de ampliar mercados, donde la población es cada vez más consumista y el deterioro de los recursos naturales aumenta, como consecuencia se manifiestan los diferentes problemas ambientales. Es así como empieza a surgir una gran preocupación por el cuidado y conservación de los recursos naturales, sin embargo existe una contradicción cuando se dice que existe desarrollo en determinado lugar porque este se da a costa de la depredación de los recursos entonces podemos decir que no entra en la lógica de la sustentabilidad.

El desarrollo sustentable es un ideal de desarrollo con equidad, que al mismo tiempo busca ser ecológicamente sano, sin embargo existen contradicciones, porque al mismo tiempo que una sociedad necesita cuidar sus recursos y conservarlos también se requiere hacer uso de ellos para poder sobrevivir, entonces se puede decir que necesita de la naturaleza para sus actividades productivas, y si quiere mejorar esas condiciones requiere de alguna manera hacerlo a costa de ella, tal vez han surgido actividades que generan menor impacto negativo a la naturaleza pero no dejan de generar ese impacto y al mismo tiempo de deteriorar los recursos.

Dentro del nuevo modelo de desarrollo económico podemos ubicar al desarrollo local, el cual pretende que el desarrollo se genere desde dentro es decir que a partir de los recursos endógenos, de la población se empiece a generar diversas actividades que beneficien a la población haciendo un uso racional de sus recursos.

Arocena (2001), plantea como una nueva interpretación, la justificación empleada por los dueños del capital económico para su intervención y manejo de los recursos de las comunidades rurales, destacando que la política del desarrollo local combina las inversiones e iniciativas de los actores locales y externos, para la mejora del conocimiento y la retroalimentación de las experiencias obtenidas de las organizaciones, señalando la 
importancia de la creación de nuevas empresas, la introducción y adaptación de nuevas formas de producción, que puedan generar cambios en el entorno cultural, favoreciendo así los procesos de industrialización endógena, en donde es necesario la existencia de esquemas culturales flexibles abiertos al destino que la globalización haga de sus recursos.

De ahí la importancia de que el desarrollo se de desde dentro; es decir que las propuestas de desarrollo surjan de las organizaciones de las poblaciones, del adecuado uso de los recursos, de la cooperación, y sobre todo de tener un objetivo común que busque el beneficio de la comunidad, que permita el verdadero desarrollo en estas comunidades, haciéndolas mas competitivas, y al mismo tiempo fortalecer su economía, porque si ellos permiten la intervención de actores externos a la comunidad los beneficios no se verán reflejados, seguirá el dominio de aquellos actores que tiene el poder económico y que toman decisiones sobre los recursos ajenos.

El desarrollo local es un complejo proceso de concertación entre los agentes - sectores y fuerzas - que interactúan dentro de los límites de un territorio determinado con el propósito de impulsar un proyecto común que combine la generación de crecimiento económico, equidad, cambio social y cultural, sustentabilidad ecológica, enfoque de género, calidad y equilibrio espacial y territorial con el fin de elevar la calidad de vida y el bienestar de cada familia y ciudadano o ciudadana que viven en ese territorio o localidad. Más aun, implica concertación con agentes regionales, nacionales e internacionales cuya contribución enriquece y fortalece ese proceso que tiene una lógica interna, que avanza de manera gradual pero no mecánica ni lineal, que le da sentido a las distintas actividades y acciones que realizan los diferentes actores (Enríquez, 1998).

Como lo menciona Enríquez, la concertación entre los diferentes actores sociales que tiene que ver con el desarrollo de la comunidad, es uno de los elementos importantes para que se de el proceso de desarrollo, ya que si no existen acuerdos entre ellos, no podrán concretar proyectos que beneficien a la población y como consecuencia elevar la calidad de vida. Llevar a cabo proyectos en común es tarea difícil porque no siempre los actores están dispuestos a participar de alguna manera se puede decir que influye el aspecto cultural y social de la población, es difícil que la gente confíe, aun más cuando ya antes ha sido engañada. Sin embargo el plantear beneficios para la comunidad se pudiera despertar el interés por participar en las actividades que les dejan un beneficio y sobre todo porque es a partir de sus recursos. 
Si uno de los problemas en los países subdesarrollados es la pobreza, el modelo de desarrollo local permite dar respuesta a esa problemática al mismo tiempo que se preocupa por la utilización racional de los recursos llevando a cabo actividades acorde a las características de estos optimizando así su uso y cuidado del mismo, lo cual permite que las futuras generaciones puedan disfrutar de los recursos.

El desarrollo es un tema que seguirá siendo estudiado, y sobre todo es el objetivo de muchos países, así que cada uno intentará alcanzarlo a partir de distintas estrategias, pero realmente se quiere alcanzar el desarrollo, a costa de la explotación de los recursos y de los cambios en la naturaleza que al mismo tiempo amenaza a la humanidad a su propia existencia, a costa de que se quiere lograr el desarrollo, como se puede obtener la calidad de vida de la humanidad, como resolver el problema de la pobreza, como alcanzar el desarrollo y al mismo tiempo aminorar el deterioro del medio ambiente.

El desarrollo que presentan las comunidades es el resultado de las condiciones de estos lugares, tiene que ver con su historia así como las condiciones sociales, económicas, políticas y ambientales, además se tiene que considerar las capacidades colectivas de dicha comunidad, ya que a mayores capacidades, mayores posibilidades de desarrollo tienen.

\section{Capacidad Colectiva}

Es indudable el papel que juegan los actores locales en el desarrollo de sus comunidades, de ahí la importancia de reconocer las capacidades colectivas de una comunidad; por lo tanto, el desarrollo local tiene que ver con las capacidades colectivas de la región, población y comunidad, esto implica más posibilidad también para impulsar su propio desarrollo.

Las capacidades de los actores locales como lo menciona Vargas se "refiere a las posibilidades de acción, habilidades y recursos que son inherentes a los actores locales, en función de tres vertientes: la organización en torno a un objetivo en común, de mejoramiento permanente de las condiciones de vida de la sociedad local; la generación de instrumentos adecuados y útiles para conseguir el objetivo señalado, y el conocimiento y visión que mantiene el ámbito local en un contexto más amplio, que es el de la globalización" $(2006: 175)$. 


\subsection{Capacidad organizacional}

Se consideran las posibilidades de acción, habilidades y recursos de los actores locales para generar la articulación de sus iniciativas, estableciendo funciones y normas específicas de comportamiento para quienes la integran, determinadas por la existencia de un objetivo y proyecto en común, de transformación sostenida y cualitativa de las condiciones de vida de la sociedad local en que se insertan y sus expectativas de progreso, por lo tanto podemos decir que esta capacidad es uno de los ejes importantes para que un grupo social pueda dirigir su propio desarrollo.

\subsection{Capacidad instrumental}

Este término hace referencia a las posibilidades y habilidades de los actores locales para generar los instrumentos que les permitan conseguir los objetivos del desarrollo local que pretenden. Tales instrumentos están agrupados en cuatro rubros: de planeación, concertación, gestión y comunicación. Los primeros se refieren a la elaboración de proyectos que articulen los intereses de los actores locales para concretar sus objetivos; los relacionados a la concertación en ellos se establecen intereses e iniciativa de los actores locales, los de gestión los cuales les permiten acceder a recursos e instancias político- administrativas y privadas necesarias para la consecución de sus objetivos y finalmente la comunicación ya que hace posible la difusión de los logros alcanzados, así como la retroalimentación del proyecto.

\subsection{Capacidad sistémica}

Se refiere a las posibilidades y habilidades de los actores para entender su posicionamiento en un contexto más amplio, que incluye los ámbitos regional, estatal e internacional, es decir, alude al conocimiento e interpretación que hagan del complejo esquema de factores y relaciones que inciden en la configuración de la sociedad y economía local (Vargas , 2006).

\section{Desarrollo Turístico y Capacidades Colectivas en San Juan de las Huertas}

Actualmente el turismo bajo la modalidad de turismo alternativo en comunidades rurales, es considerado una de las actividades económicas que generan un bajo impacto al medio ambiente y al mismo tiempo permite mejorar la calidad de vida de las localidades que cuentan con recursos naturales susceptibles de aprovechamiento racional para el turismo ( Paré 2003 , Daltabuit et al., 2002, Monterroso 2003, Zizumbo 2006, 2007, 2008, Pérez 2006, Enríquez 2007). Es un tema reciente que interesa a gobiernos, empresas, ONG y académicos porque 
ven en esta actividad posibilidades de vincularse con el medio rural. Sin embargo no todas las comunidades tienen la capacidad de aprovechar sus recursos y generar al mismo tiempo alguna actividad que les permita obtener algún beneficio, como lo es el turismo.

El turismo se presenta por una parte como una propuesta de mejorar las condiciones de vida de la población rural y por otra como una estrategia de conservación. Las posibilidades de que la comunidad de San Juan de las Huertas cuente con cierto nivel de organización para poder llevar a cabo proyectos turísticos en su comunidad impulsados por los gobiernos estatales, municipales y locales ha sido limitado para su desarrollo

De acuerdo a los resultados de la encuesta se pudo comprobar que la organización ejidal ha sufrido debilitamiento debido a que el $90 \%$ de los ejidatarios no creen en su organización, ya que señalan que sólo los dirigentes se han beneficiado de sus puestos al tomar decisiones, sin que exista equidad en el reparto de los beneficios para la población en general.

Otra dificultad que se presenta en la capacidad organizacional es que con los cambios generados con la reforma agraria han permitiendo la venta de tierras, ingresando a la organización nuevos ejidatarios que no son de la comunidad, han comprado estas tierras convirtiéndose así en ejidatarios, quienes no se sienten integrados a la comunidad por lo que desconocen los problemas, el uso, manejo y aprovechamiento de los recursos provocando una mayor división en la organización, así como apatía a impulsar proyectos dentro de la comunidad.

A los ejidatarios la riqueza natural con la que cuentan: bosques, minas de arena, valles, tierras agrícolas no les han significado beneficios de manera equitativa tanto en la contribución de ingresos generados, ni en la eliminación de prácticas que destruyen el medio ambiente. Lo que ha provocado una indiferencia en la toma de decisiones y en la asistencia a las Asambleas Generales de Ejidatarios en donde se toman los acuerdos. No existe un liderazgo democrático al no permitir movilizar a los integrantes de la comunidad y lograr concertaciones a largo plazo. Privan los valores individuales, las actitudes y sensibilidad frente a las diversas formas de inequidad social. Los ejidatarios solo consideran que forman parte de la organización, sin que ello les beneficie.

La situación económica por la que atraviesan los ejidatarios no permite consolidar la organización, la cual requiere de dedicación de tiempo y en algunos casos de dinero para poder resolver los problemas de invasiones de tierras, de litigios por el manejo de los recursos, etc. Al buscar otras fuentes de ingresos que no son de la agrícultura porque desde 
varias décadas atrás no da para la subsistencia de la familias y el poco apoyo de los representantes ejidales de impulsar proyectos productivos democráticos, participativos y equitativos ha hecho que los ejidatarios no se organicen para impulsar su Ejido.

La falta de identidad a la organización hace que no tengan confianza y que no mantegan redes de reciprocidad mutua entre los ejidatarios. Por lo que carecen de visiones a futuro, propuestas, actiudes y normas claras para impulsar el desarrollo en la comunidad. Los sistemas de colaboración se han ido perdiendo, las relaciones entre personas e instituciones son verticales y clientelares, hay gran concentración del poder y de la riqueza, las distinciones aunque no son tan marcadas existen y se conocen.

La organizacón ejidal tiene serios problemas, por la falta de legitimidad y participación efectiva de sus integrantes, debido a que por años no se les tomó en cuenta y ahora que necesitan de ellos tienen dificultades para que se integren y confien en sus representantes. Se pudó constatar que dentro de la organización ejidal no existe una división de tareas, es decir sólo los que se encuentran en la directiva de la organización ejidal se dividen las tareas las cuales no estan bien definidas, las realizan de acuerdo a las necesidades del ejido y en el momento que se presenten deciden que realizar, los demás integrantes del ejido no realizan alguna actividad, a menos que sean convocados.

La participación en la organización ejidal ha sido limitada a ejidatarios con su título, así podemos encontrar a hijos de ejidatarios que a pesar de tener el derecho a participar en ella no se los permiten por no contar con el documento que lo acredite, es uno de los motivos por los que el interés de los hijos de los ejidatarios en participar en la organización ejidal es baja.

El capital humano ejidal de la comunidad el cual ha estado constituido por la habilidad y el talento individual de los seres humanos que habitan la comunidad, su capacidad de ser, hacer y decidir por sí mismos; su grado de capacitación, el grado de reflexión crítica y el nivel de educación para concebir, diseñar y ejecutar proyectos y planes de desarrollo para la comunidad, así como los niveles de liderazgo para dirigir el desarrollo comuntario considerando las experiencias y conocimientos ancestrales, así como los valores éticos han sido corrompidos por agentes externos, logrando debilitar la capacidad intrumental de la organización ejidal.

Se trata de un organización comunitaria débil que se esta dejando morir, la poca asistencia de los ejidatarios a las asambleas, la falta de renovación de sus integrantes, así como la toma de decisiones verticales por los dirigentes hace que la percepción que tienen los ejidatarios sea de 
que la organización no ha tenido logros, no concreta acciones y no ha obtenido beneficios; lo anterior se debe a la desconfianza de los integrantes de la organización, falta de capacidad de los dirigentes, apatía, avaricia por parte de los dirigentes, engaño por parte de las autoridades promotoras externas a la comunidad, falta de mecanismos de integración entre el grupo ejidal, y a la falta de participación de la comunidad.

Las intervenciones internas y externas para impulsar proyectos turísticos y con ello la conservación de los recursos naturales por parte la comunidad ha sido limitada, así como los múltiples programas gubernamentales de desarrollo tipo asistencialista que han llegado a San Juan de las Huertas, ofreciendo el apoyo de infraestructura turística a cambio de sus recursos naturales (el bosque y minas) no han resuelto a corto, mediano y largo plazo las condiciones de pobreza de la población, ni el deterioro ambiental de sus recursos. Lo que ha sucedido es crear mayor dependencia hacia las instituciones y debilitar las organizaciones comunales internas.

La consolidación de un grupo o grupos dentro del ejido ha permitido solamente logros de acciones para beneficio de ellos mismos, por lo que los ejidatarios consideran que su organización ejidal de San Juan de las Huertas no esta consolidada.

Los ejidatarios no tienen claridad sobre las acciones que han desarrollado sus dirigentes y qué ha sucedido con sus recursos, desconocen qué pasa internamente, de lo que más se habla es de los problemas que se tienen en cuanto a límites con otras comunidades, pero sobre los beneficios que han obtenido con el manejo y aprovechamiento de sus recursos naturales (bosque, agua, minas) no saben. Por lo que es claro que no hay transparencia en cuanto a la forma en que se vienen manejando sus recursos y los ingresos que se obtienen de éstos y cual es su destino.

En cuanto a la capacidad que tiene la organización para llevar a cabo proyectos comunitarios es limitado, ya que de ello depende en gran parte el beneficio que puedan obtener como grupo.

El poblado de San Juan de las Huertas como ya hemos señalado cuenta con bastos recursos naturales (bosque, agua, minas de arena, valles y un área natural protegida en la que se encuentra el Parque de Los Venados). Sus recursos han sido objeto de intereses externos a la comunidad, lo que les permitió vincularse desde hace tiempo atrás con instituciones y políticos que les ofrecían mejorar su comunidad a cambio de la exploatación de los mismos. 
La organización ejidal no se ha visto limitada en su capacidad sistémica para vincularse con actores externos a la comunidad, por un lado le ha permitido la obtención de benefícios para el grupo representante a partir del impulso de diversos proyectos; esas relaciones les permitieron durante años beneficiarse a costa de la comunidad. Sin embargo, los dirigentes actuales buscan limpiar y rescatar a la organización ejidal para que los recursos con los que cuentan sean aprovechados de manera sustentable y que permita mejorar las condiciones de vida de la población ejidal.

Los proyectos productivos impulsados en la comunidad no tuvieron el consenso de los integrantes de la Asamblea General de Ejidatarios y no tuvieron como finalidad que los incentivos económicos ligados a la inserción de un producto o servicio en el mercado impulsara una reconversión productiva que transitara de un modelo de sobreexplotación de los recursos a otro que incorporara cada vez más elementos de sustentabilidad a la economía local. Esto implicaba el fortalecimiento de las capacidades locales lo cual no sucedió, porque en su lugar se fueron diluyendo, al no incentivar la participación y transparencia en la toma de decisiones, lo cual provoco la total desconfianza en los grupos que los representaban.

Los proyectos productivos generados en la comunidad de San Juan de las Huertas han sido impulsados por actores externos, ya sean las instituciones públicas o privadas, los proyectos no han sido por iniciativa de la comunidad, por lo cual no han tenido su legitimidad y credibilidad. La propia condición de la comunidad en cuanto a sus aspectos sociopolíticos ha cambiado, lo que ha provocado el desinterés, desconfianza u oposición a estos proyectos.

Es importante señalar que la capacidad sistémica para impulsar proyectos en la comunidad queda en manos de los dirigentes ejidatarios y que es para su propio beneficio y que los demás integrantes no tienen conocimiento sobre lo que sucede en su organización solo tienen una lejana idea de lo que pasa, reconociendo que ha habido proyectos pero deconocen los beneficios de éstos y las causas de los fracasos de los mismos, quienes han participado e impulsado este tipo de proyectos, con ello podemos determinar por una parte la autonomía de la organización y por otro lado la capacidad de relacionarse con otras instituciones públicas o privadas para concretar proyectos.

Dentro de los proyectos que se han mencionado se habla del turismo, sin embargo éste no ha tenido el impulso suficiente para desarrollarse, aun cuando está en manos de los ejidatrios el poder impulsar la actividad a partir de sus recursos naturales e infraestructura con los que cuentan dentro del ejido; el impulso de acciones para el turismo depende de las autoridades en 
este caso del comisariado ejidal, a partir de sus ideas plasmadas en proyectos e incluso de la importancia que estos le den a la actividad turística.

Lo que sorprende de la investigación, es que teniendo recursos naturales para el desarrollo del turismo, no todos los ejidatarios reconocen las posibilidades de participar en estos espacios por lo que la mayoría no están involucrados en la actividad turística. Ellos señalaron que no han visto en el turismo una alternativa de mejorar sus condicione de vida, sino que han optado por diversificar sus actividades económicas, sus intereses y estilo de vida, pocos son los que se han involucrado en la actividad.

Es importante para poder impulsar algún proyecto conocer los recursos con los que cuenta la organización ejidal, ya que a partir de éstos se pueden organizar y planear actividades para impulsar la actividad turística, de acuerdo a la visión de los ejidatarios.

La situación que se visualiza es que existe acciones desarticuladas entre las instituciones no lograndose consolidar las estrategias. Es importante comprende que las interrelaciones entre el contexto han sido de gran vulnerabilidad, al no considerar las instituciones los problemas internos dentro de la propia comunidad y en el interior de la organización ejidal

El turismo puede ser una buena alternativa de desarrollo de la comunidad, siempre y cuando los ejidatarios tengan conciencia de que el recurso natural con el que cuentan es primordial para impulsar uno o varios proyectos, que se cuenta con el recurso humano pero hace falta de una organización fuerte basada en relaciones sociales de confianza, honradez y respeto, además de consolidar un grupo ya sea a través de una cooperativa que se encargue del mantenimiento, vigilancia y protección tanto de los recursos naturales como del turismo, un grupo permanencte que dedique tiempo completo en la promoción de las actividades turísticas que decidan ofrecer.

La falta de autonomía en los proyectos turísticos impulsados en la comunidad no ha permitido fortalecer al núcleo ejidal. El hecho de centralizar las funciones en las autoridades de los ejidos trajo consigo la indiferencia, desconfianza y falta de participación de los ejidatarios, provocando con ello un mayor empobrecimiento y marginación, lo cual se ve reflejado en la gran migración de los jóvenes hijos de ejidatarios en busca de empleo, ya que la comunidad ha sido incapaz de proporcionar a las nuevas generaciones alternativas de sobrevivencia, aunque cuente con una riqueza natural como son sus bosque, valles y áreas naturales. 
El impulso de grupos, asociaciones o cooperativas de participación voluntaria puede ser efectivo siempre y cuando las propuestas vengan de las bases, sean ellos los propios ejidatarios los que impulsen los proyectos, sin que vengan de agentes externos para que sean ellos los que decidan sobre los mecanismos internos en la toma de decisiones y de la propia organización.

Las posibilidades de acción colectiva para el turismo en el ejido de San Juan de las Huertas, han sido casi nulas, debido a varios factores, en primer lugar el Parque de los Venados, que se constituye como un recurso forestal el cual cuenta con un extraordinario paisaje, así como áreas para recrearse, no se ha constituido como un recurso importante para la subsistencia de los ejidatarios, ya que no han obtenido beneficios tangibles como producto de la actividad turística; además el hecho de reactivar la actividad les implicaría costos, y como organización no cuentan con los recursos económicos para invertir lo han dejado.

En segundo lugar para que se pueda dar la acción colectiva es necesaria la participación de los integrantes de la organización ejidal, sin embargo esta no se ha dado, existe baja participación lo cual no permite que los integrantes del ejido conozcan, opinen y propongan alternativas para el aprovechamiento de su recurso; otro de los factores que tiene que ver con la acción colectiva es la confianza que existe entre los miembros del grupo, esta se ha perdido debido a los malos manejos de las administraciones pasadas, que han explotado sus recursos y no se ha visto beneficiado el ejido, esa falta de confianza entre los miembros no permite que pueden actuar como un solo grupo, y se vallan perdiendo sus intereses en común anteponiendo sus intereses individuales.

La falta de compromiso con el ejido, no permite que se realice una programación de actividades dentro del parque, esto es como consecuencia de la falta de interés por el mismo. Si bien es cierto actualmente existe un interés por parte de las autoridades ejidales por rescatar el parque, sin embargo el poco de interés por parte de los ejidatarios, la desconfianza y falta de responsabilidad por parte de los integrantes del ejido, no les ha permitido proponer acciones para desarrollar la actividad turística.

En cuanto a los instrumentos de planeación, la organización no ha generado proyectos para el parque de los Venados, que permita por un lado integrar los intereses de los ejidatarios y por otro su aprovechamiento de manera sustentable, esto se debe a que no existe interés por desarrollar la actividad, y por otra por la falta de conocimientos sobre la misma, lo cual no les permite proponer alternativas para mejorar las instalaciones, el servicio o proponer las 
mismas actividades dentro del parque. Como consecuencia de una nula planeación, no existen objetivos por parte de las autoridades ejidales, ni estrategias para mejorar la actividad, falta definición de responsabilidades y por lo tanto de tareas. La falta de continuidad a las actividades entre una directiva y otra del comisariado ejidal no permite dar un seguimiento y mucho menos la evaluación de las acciones para el turismo, esto no ha permitido identificar los aciertos o los errores de las administraciones pasadas, para que de ahí partieran las nuevas administraciones.

No se han fortalecido los instrumentos de concertación, entre los integrantes del ejido, debido a la poca participación, falta de compromiso por parte de los ejidatarios y sobre todo a la división de intereses como grupo; esta división de interés se da porque piensan en el beneficio de manera individual, buscando otras alternativas para subsistir y el turismo no ha sido uno de sus intereses, por lo tanto no han llegado a acuerdos para impulsar el turismo.

La habilidad de gestionar apoyos ante instancias político-administrativas y privadas para el logro del desarrollo del turismo no se ha dado, principalmente porque el turismo no ha sido su interés, de ahí que ellos han gestionado apoyos para otras actividades como lo es el bosque, han solicitado apoyos para reforestar el bosque y lo han logrado, sin embargo en cuanto a la actividad turística solo han obtenido algunos señalamientos para el Parque de los Venados y un curso para preparación de alimentos el cual quedo inconcluso. Queda claro que si no existe el interés por parte de la organización ejidal difícilmente lograrán obtener apoyos, es importante que identifiquen las instancias a las que pueden acudir a solicitar estos apoyos.

La capacidad que tiene la organización ejidal para vincularse con actores externos a la comunidad permiten obtener beneficios para el grupo a partir de proyectos; esas relaciones le permiten vincularse con otros actores con una visión diferente y conocer otras formas de organización.

Sin embargo en el ejido existe una vinculación con instancias agrarias, e incluso con aquellas que están relacionadas con el bosque, pero falta vincularse con instituciones que pueden apoyar la actividad turística, es decir saben vincularse, pero existe un desconocimiento sobre los apoyos que pueden obtener, a partir de la actividad turística. Este desconocimiento ha limitado su visión para desarrollar el turismo en el parque, si bien es cierto ellos pueden solicitar apoyos para mejorar las instalaciones del parque. La vinculación con instancias internacionales es nula, así como el acceso a nuevas tecnologías, ya que no lo aplican a las actividades económicas actuales. 


\section{Conclusiones}

San Juan de las Huertas, comunidad marcada por los hechos históricos de la misma, siendo esta una comunidad ejidal, define su propio desarrollo a partir del uso de sus recursos naturales como es el bosque, agua y tierras de cultivo; sin embargo a pesar de que tuvo recursos naturales importantes, no logró beneficiarse con el aprovechamiento de sus recursos.

La organización ejidal de San Juan se encuentra dividida, por intereses particulares, este hecho se ha dado a través del tiempo, sin embargo los efectos o repercusiones se ven reflejadas en su vida cotidiana, o incluso en su mismo desarrollo. Ya que podemos identificarlo como un organismo en donde sus miembros no están agusto, existe desconfianza hacia las autoridades, cada uno ve por sus propios intereses y no lo hacen de manera colectiva.

La Comunidad ejidal de San Juan cuenta con instalaciones dentro del parque de los Venados, desde hace más de 30 años, sin embargo las condiciones en que se han organizado para la actividad no han sido las apropiadas ya que no han logrado el desarrollo de la actividad turística, no se ha dado un seguimiento de la misma, y han dejado que se de por sí sola.

En relación a las capacidades del ejido se pudo identificar en relación a la capacidad organizacional que esta no existe debido a que no tiene definido un objetivo en común, de ahí que cada uno de los integrantes de la organización, ve por sus propios intereses y busca la manera de satisfacer sus necesidades sin tener que apoyar de la organización ejidal, la falta de un objetivo en común es un factor que determina la organización colectiva; otro indicador es la falta de proyectos en común, en este caso la falta de proyectos turísticos impulsados por ellos mismos.

Las capacidades instrumentales que tiene la población tiene que ver con la existencia de instrumentos de planeación los cuales no existen para la actividad turística, existen para la actividad forestal, sin embargo son unos pocos los que participan en ello; otro indicador son los instrumentos de concertación sin embargo uno de los problemas que tienen en relación a la concertación es la falta de participación, en relación a la gestión son pocos los integrantes de la organización que saben gestionar sus proyectos siendo estos principalmente relacionados con el bosque, con las tierras, pero o con el turismo; otro factor muy importante para el desarrollo del turismo es la existencia de instrumentos de comunicación el cual propicie un trabajo armónico sin conflictos, sin embargo la comunicación no ha sido tan efectiva entre los integrantes la organización ejidal de ahí los malos entendidos y sus conflictos. 
La capacidad sistémica que tienen la organización es casi nula, ya que ellos no tiene los conocimientos ni percepción en relación a el contexto internacional, ni la existencia de vínculos con actores locales de otras localidades, regiones o a nivel internacional, lo cual ha limitado un poco el desarrollo, sus vínculos son de manera interna sólo el presidente Ejidal es el que tiene las posibilidades de relacionarse con otros actores locales de comunidades cercanas.

A partir de las capacidades de una comunidad se puede definir las posibilidades de desarrollo, en este caso el bosque como uno de los recursos naturales con los que cuenta el ejido y no ha sido aprovechado para beneficio de la comunidad, la actividad turística es una de las actividades que se pueden desarrollar en el parque, esta modalidad del turismo no requiere de tanta inversión y es muy fácil de que la comunidad se integre a las actividades dentro del parque.

Existen los recursos naturales para impulsar el turismo, cuentan con una infraestructura para poder proporcionar servicios, pero no existen capacidades colectivas, han perdido esa capacidad a lo largo del tiempo debido a conflictos dentro del mismo grupo, la desconfianza, la falta de equidad, la baja participación de los miembros genera conflictos.

Es importante reconocer que se pueden fortalecer las capacidades colectivas y hay que aclarar que al hablar de capacidades colectivas nos referimos al saber trabajar por un objetivo en común y no es únicamente la suma de todos los esfuerzos; el fortalecimiento de estas capacidades lleva tiempo sin embargo es un proceso que se puede lograr a partir del esfuerzo de todos.

\section{Bibliografía}

Arocena, José. 2001. Globalización, integración y desarrollo local. En: Transformaciones globales, instituciones y políticas de desarrollo local, Antonio Vázquez Barquero y Oscar Madoery (Comps). Homo Sapiens Ediciones, Buenos Aires.

Alberto Villavicencio, Ángeles. 1997. Propuesta de aprovechamiento ecoturístico-recreativo del ejido San Juan de las Huertas, Parque Nacional Nevado de Toluca. Tesis de Maestría, Facultad de Filosofía y Letras, UNAM.

Boiser, Sergio. 2001. "Desarrollo (local). ¿De qué estamos hablando?” en: Transformaciones globales, instituciones y políticas de desarrollo local, Antonio Vázquez Barquero y Oscar Madoery (Comps). Buenos Aires, Homo Sapiens Ediciones.

Centro de Estudios Agropecuarios. 2001. Turismo Rural y Ecoturismo, Grupo Editorial Iberoamericana. México. 
Chávez, Araceli y Cisneros Guillermo. 2004. Análisis geográfico: la actividad agrícola y su relación con las condiciones de vida de la población en el ejido de San Juan de las Huertas, Zinacantepec. Tesis de Licenciatura. Facultad de Geografía, UAEM.

Coraggio, Jose Luis. 2003. La política social y economía del trabajo. Zinacatepec, México, Miño y Dávila/ Colegio Mexiquense A.C.

www.ubared.ungs.edu.ar.

Daltabuit, Magali et al. 2002. Ecoturismo y Desarrollo Sustentable. México UNAM/CRIM.

Enríquez Villavicencio, Alberto. 1998. Desarrollo sustentable y desarrollo regional/local en el Salvador, alternativas para el desarrollo. Revista Sociedad Civil No. 7. DEMOS, México.

Enríquez Pérez, Dulce. 2007. Desarrollo Local a partir del turismo en la comunidad de San Pedro Atlapulco, Municipio de Ocoyoacac, Estado de México, Tesis de Licenciatura. Facultad de Turismo, UAEM.

Marsiglia, Javier y Pintos, Graciela. 1997. La Construcción del Desarrollo Local como desafio metodológico en el Programa de Desarrollo Local, Editorial CLAH, Montevideo, diciembre.

Monterroso Salvatierra Neptalí. 2003. El poder local: premisa del desarrollo rural. El caso de la comunidad de San Pedro Atlapulco, México. Segundo Seminario Internacional, procesos y mecanismos de participación y construcción de capital social para un efectivo manejo de los recursos naturales, Valdivia, Chile.

Paré Luisa y Elena Lazos. 2003. Escuela rural y organización comunitaria: Instituciones locales para el desarrollo y el manejo ambiental. Instituto de Investigaciones Sociales UNAM/Plaza y Valdés, México.

Pérez Ramírez, Carlos Alberto. 2005. El capital social y la actividad turística en San Pedro Atlapulco: Bases para el desarrollo rural. Tesis de Licenciatura, Facultad de Turismo. UAEM, México.

Preston. P. W. 1999. Una introducción a la Teoría del Desarrollo, Siglo XXI Editores, México.

Quintanilla Montoya Ana Luz, Rojas Caldelas, Rosa Imelda, Zizumbo Villarreal Lilia y Sergio Larios. 2004. Building up the future: Participative design of a rural development plan for the coastal communites of the arid regions of Baja California, México. En: Libro Coastal Envirnoment, Publicado en United Kingdome por Wessex Institute of Science and Technology.

Vargas Castro, José Alejandro. 2006. El desarrollo local en el contexto de la globalización. Editorial INAP, México.

Vázquez Barquero Antonio. 1988. Desarrollo local Una estrategia de creación de empleo, Ediciones Pirámide, Madrid.

. 2001. Desarrollo endógeno y globalización. En: Transformaciones globales, instituciones y $\overline{\text { politicas }}$ de desarrollo local, Homosapiens Ediciones, Rosario Argentina.

Zizumbo Villarreal Lilia. 2006. El turismo en Bahía de los Ángeles. En: Quintanilla Montoya Ana Luz (Coordinadora) Construyendo el futuro: Visiones para un desarrollo rural sustentable en las comunidades de Baja California. Universidad Autónoma de Baja California/Porrúa.

2007. El turismo en comunidades rurales: práctica social y estrategia económica. Tesis doctoral. Universidad Nacional Autónoma de México.

. y Neptalí Monterroso Salvatierra. 2008. Turismo Rural y Desarrollo Sustentable. Universidad Autónoma del Estado de México, Toluca.

Recebido em: 24/11/2008

Aprovado em: 08/05/2009 